\title{
Heterogeneities in Aging Models of Granular Compaction
}

\author{
Jeferson J. Arenzon \\ Instituto de Física, Universidade Federal do Rio Grande do Sul \\ CP 15051, 91501-970 Porto Alegre RS, Brazil
}

Received on 4 August, 2005

\begin{abstract}
Kinetically constrained models (KCM) are systems with trivial thermodynamics but often complex dynamical behavior due to constraints on the accessible paths followed by the system. Exploring these properties, the KobAndersen (KA) model was introduced to study the slow dynamics of glass forming liquids and later extended to granular materials. In this last context, we present new results on the heterogeneous character of both in and out of equilibrium dynamics, further stretching the granular-glass analogy.
\end{abstract}

Keywords: Granular material; Dynamical heterogeneities; Glass transition

\section{INTRODUCTION}

In recent years, the analogy between structural glasses and dense granular systems has become deeper and extensively explored [1-25]. As temperature is lowered, or density increased, respectively, both systems undergo a glass, or jamming, transition, where the relaxation times dramatically increase. Yet, in spite of all evidence of phenomenological similarity, the two systems are fundamentally different, for example, in the length scale of its components (molecules versus macroscopic particles) and the role of thermal energy (none, in the case of granulars). Because the thermal energy is too small to induce movement in these macroscopic particles, energy should be externally supplied, for example, by vibrating, tapping, shearing, rotating, etc, the system, in order for a granular system explore its configurational space.

Recently, the role of dynamical heterogeneities in the complex dynamics of glassy systems has been addressed [26-29]. The glass transition seems to be purely dynamical, with no increasing static correlation length as the system approaches the transition, differently from the usual critical slowing down. Thus, the increase in relaxation times seems to be related to a diverging dynamic correlation length [30-36], associated to the increasing number of particles whose displacements become dynamically correlated, the heterogeneities, that develop during the evolution of the system. In Ref. [37] we showed, at the level of a simple, kinetically constrained model [38], that dynamical heterogeneities seem to play the same role in granular systems as they do in structural glasses, with an increasing length scale as the system approaches the jamming transition. This has also been observed in other recent works [20, 25, 39-41]. However, the precise role played by these structures and the associated lengths, on the dynamics of granular and colloidal systems, is yet to be understood.

The jamming transition has also been studied in connection with the concept of dynamically available volume (DAV) [42, 43]. Apart from having enough nearby empty space [44], a particle is mobile if, for the particular type of model considered here, the displacement is allowed by the kinetic constraints. Empty sites that are able to receive a neighboring mobile particle are called holes. Holes can be classified as either connected or not, the former being those that, by allowing a particle to jump into it may eventually facili- tate the movements of all particles in the system. On the other hand, nonconnected holes leave a backbone of blocked particles. Close to dynamical arrest, the density of connected holes decreases with the density of particles and is related with the inverse of the bootstrap length $[45,46]$, the average distance between two connected holes, in bootstrap percolation. In turn, this length is associated with the transport properties of the system [47-49].

Here we make an initial attempt of studying how these holes behave when the system is externally driven and falls out of equilibrium, in particular, their role during the compaction regime of granular systems, what is connected with the voids distribution measured experimentally [50]. Although we do not distinguish at this stage connected from disconnected holes, this would be important in order to fully understand the microscopic compaction mechanism.

\section{KOB-ANDERSEN MODEL}

The Kob-Andersen model [51] is one of the simplest models describing the complex dynamics of glassy and granular systems. It consists of a lattice gas of $N$ particles, each site being either empty or occupied by one particle, with no static interactions between them, i.e., $\mathcal{H}=0$. In addition, a kinetic constraint [38] should be satisfied in order to allow the displacement of a particle to an empty neighboring site: there should be fewer than $m$ occupied nearest neighbors before and after the move. This kinetic rule is time-reversible and detailed balance is satisfied. If the constraint is obeyed, the particle is said to be mobile and the companion vacant site is said to be a hole. At high densities, the dynamics slows down because the reduced free-volume makes it harder for a particle to satisfy the dynamic constraints. These constraints were introduced to mimic the cage effect where, due to geometrical effects, the displacements of the particles are hindered by their neighbor particles.

Although on hypercubic lattices this model presents a jamming transition only at full occupancy [48], with a super Arrhenius behavior of the relaxation time, for finite lattices the bootstrap length may become larger than the system size $L$. Thus, for a finite sample, the system may be frozen due to the lack of connected holes, implying the existence of a dynam- 
ical critical density $\rho_{\mathrm{c}}(L)<1$, slowly increasing with $L$. At this point, it is observed that the diffusivity falls to zero as a power law [51], $D(\rho) \sim\left(\rho_{c}-\rho\right)^{\phi}$, with $\rho_{c}$ depending, in addition, both on the lattice geometry and on the kinetic constraint $m[37,51,52]$. Assuming such a power law form for the diffusivity, finite systems, both with and without gravity, are well described, qualitatively and quantitatively, by a nonlinear diffusion equation [37, 53, 54].

Since much of dynamical properties of both structural glasses and dense granular materials are dictated by steric constraints, we have generalized [22] the Kob-Andersen model by including a gravitational field. The Hamiltonian now has a one body term, $\beta \mathcal{H}=\gamma \sum_{i} z_{i} n_{i}$, where $n_{i}=0,1$ is the occupation variable of the $i$-th site whose height is $z_{i}, \gamma=m g / k_{\mathrm{B}} T$ is the inverse gravitational length and $g$ is the constant gravitational field acting in the downward direction. We follow a continuous vibration dynamics, assuming that the random diffusive motion of particles, produced by the mechanical vibrations of the box, can be modeled as a thermal bath of temperature $T$. The particles satisfying the kinetic constraints may always move downwards while upward movements are accepted with a probability $x=\exp (-\gamma)$, related to the vibration amplitude. Particles are confined in a closed box of bcc structure, with periodic boundary conditions in the horizontal direction. We set the constraint threshold at $m=5$. As the Markov process generated by the kinetic rules is irreducible on the full configuration space [22], the static properties of the model are those of a lattice-gas of non-interacting particles in a gravitational field, and these can be easily computed.

\section{HETEROGENEITIES}

Several measures for quantifying spatial heterogeneities have been introduced for kinetic models [38]. In particular, this issue was recently investigated in the KA model $[49,55]$ without gravity using fourth-order correlation functions [45, 46]. In Ref. [37] these were extended to include the non-zero gravity case. In Fig. 1 we plot the dynamical nonlinear response

$$
\chi_{4}(z, t)=N\left(\left\langle q^{2}(z, t)\right\rangle-\langle q(z, t)\rangle^{2}\right)
$$

where $N$ is the number of sites in the computation, $q(z, t)=$ $C(z, t) / C(z, 0)$ and

$$
C(z, t)=\frac{1}{N} \sum_{i} n_{i}(t) n_{i}(0)-\rho(z, t) \rho(z, 0) .
$$

For all vibrations considered, the system is in the fluid phase and is able to achieve the asymptotic state very fast. Notice that, differently from previous works, here we measure these quantities separately around each layer of the system (to improve the averages, $i$ runs over all sites in the $z, z-1$ and $z+1$ layers). In other words, we are probing horizontal, microscopic heterogeneities, not the macroscopic ones due to the intrinsicly inhomogeneous vertical profile. Analogously to what happens in the KA model without gravity and in other glassy

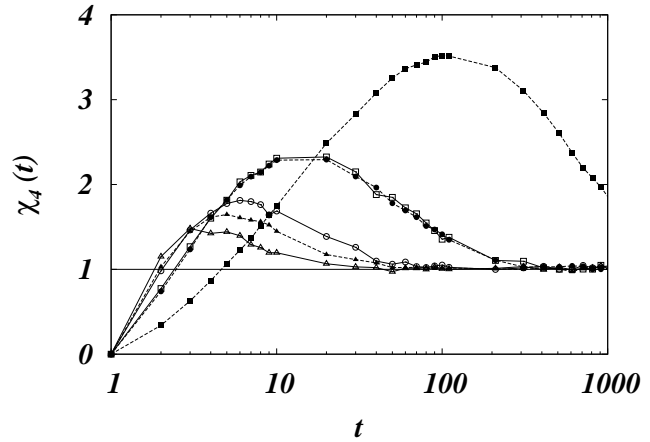

FIG. 1: Dynamical response, eq. 1, as a function of time (in MCS) for different vibrations: $\mathrm{x}=0.92$ (filled symbols) and 0.94 (empty symbols). Different symbols stand for different heights: $z=5,10$ and 15 (square, circle, triangle, respectively). The line is the asymptotic $\chi_{4}=1$ behavior [38]. Notice in the figure the presence of two very close curves: they correspond to different vibrations and heights, but their density is the same to within numerical accuracy. From [37].

systems, the peak is shifted to higher times and gets larger as the density increases (the lower is $z$, the greater is the density), which is an indication of cooperative dynamics, as larger clusters have more difficulty to respond to a perturbation. As these measures are done for vibrations above the apparent jamming threshold, the behavior of the system should not be affected by the finite size shortcomings discussed above and one is able to obtain the true, infinite size behavior. Indeed, the growth of the peak is compatible with the known relaxation time [48],

$$
\tau \sim \exp \exp \left(\frac{c}{1-\rho}\right)
$$

as can be seen in Fig. 2. Analogous results can be obtained also from the peak of the Kovacs hump [56] and persistence times distribution. Interestingly, $\chi_{4}$ only depends on $z$ through its local density, $\chi_{4}(z, t)=\chi_{4}(\rho(z), t)$ : Fig. 1 shows that two curves corresponding to different heights and vibrations $(z$ and $x$ ), but having almost the same density (within numerical precision), coincide.

A more direct measure of inhomogeneity comes from the mobile particles or, equivalently, the holes at different heights, without distinguishing, at this stage, between connected and non-connected holes. In the inset of Fig. 3, we show the density and holes profiles, $\rho(z)$ and $v(z)$, respectively, still in the fluid phase, as a function of $z$ for two different vibrations $x$. The density decreases with height and the stronger the vibration, the flatter is the density profile and the broader is $v(z)$, that is, the more vertically homogeneous the system is. Indeed, for $x=1$ (no preferential direction) both profiles are flat. Notice also that as $x$ decreases, the holes concentrate in the region that will form the interface once the system goes out of equilibrium. The interesting result that can be seen in Fig. 3 appears when we eliminate $z$ and plot $v(z)$ as a function of $\rho(z)$ : the data for different values of $x$ perfectly collapse onto a single master curve. Moreover, they are on top of the points for the KA model without gravity. In other words: even 


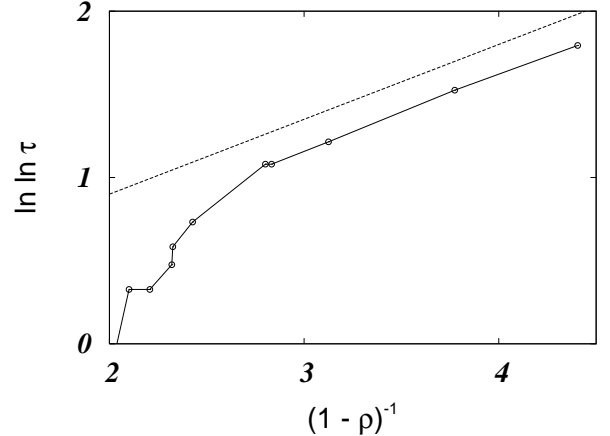

FIG. 2: Position of the peak in $\chi_{4}$. The growth is compatible with the known relaxation time [48], Eq. 3 (dashed line).

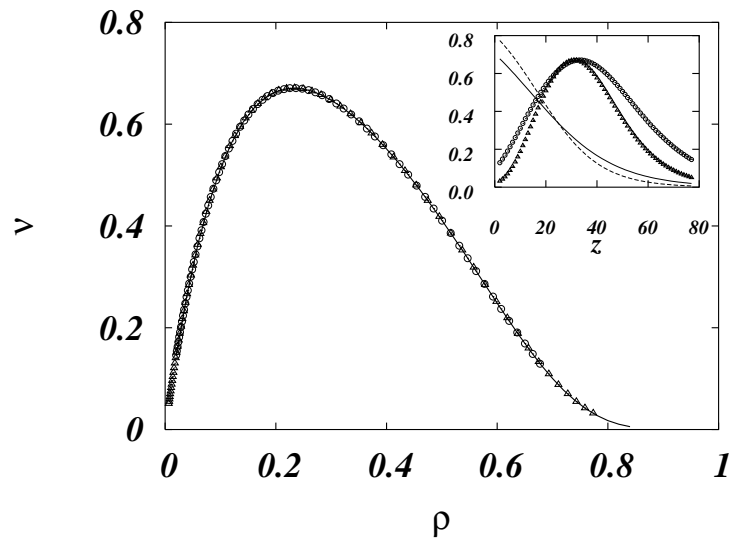

FIG. 3: Density of holes as a function of density for $x=0.92$ (triangle) and 0.94 (circle), along with the results for the KA model without gravity (line) in a bcc lattice of height $4 L$, with $L=20$. Inset: holes profile (symbols) along with the corresponding density profiles (lines). The higher $x$ corresponds to the flatter density profile and to the broader holes profile.

if the system is no longer homogeneous, the local density of holes in equilibrium only depends on the local density, and not on the whole profile, or on the vibration or the height. This, together with the analogous result for $\chi_{4}$, if general, is an important property: as some of the quantities depend only on the local density, not on the whole profile, there is further support for even simpler, one dimensional models as well as local density approximations $[37,53,54]$. $>$ From the point of view of simulation, it is a fast way to obtain, in just one simulation, the whole profile for systems without gravity.

Upon decreasing the vibration, a finite system enters in the aging regime and the density profile develops two very distinct regions [54]: an almost flat plateau at $\rho_{\mathrm{c}}$ for $z<z_{0}$ and a density decreasing region (interface) for $z>z_{0}$. The position of this plateau is size dependent and slowly increases with $L$, similar to $\rho_{c}$. Analogously, the hole profile also has two corresponding regions (see Fig. 4 and the inset): at the interface

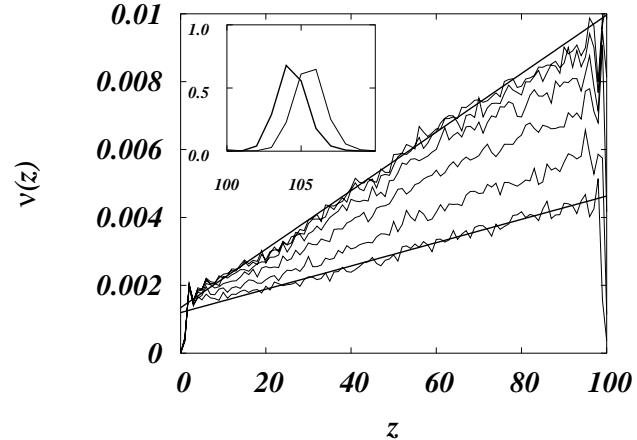

FIG. 4: Hole profile at different times (smaller time at the top). The profile is made of two clearly separated parts: $i$ ) the interface (inset) contains almost all holes in the system and moves to the left as the system becomes compact; ii) the bulk has a much smaller hole fraction, that increases linearly with height. Separating these regions we have a few layers near $z=100$ that are almost hole-free. The declivity slowly decreases with time. Notice also the difference on the vertical scales.

where most holes are localized and in the bulk, where their number is much smaller. These regions are separated by a dip with a few layers width, where there are almost no holes, corresponding to the dense layer seen in the density profile near the interface, at the top of the granular pile (this region also seems to become more localized with time), as seen in Fig. 5. At the interface, the profile is strongly peaked, and moves to the left, accompanying the interface, as the system ages (compactifies). On the other hand, in the bulk, the profile is linear, with a tangent that slowly decreases in time (although also compatible with the inverse logarithm law, a good fit is obtained with $t^{-0.25}$ ). This evaporation of holes is the direct mechanism of the compactification process. Also, because of the almost hole-free layers between the bulk and the interface, it is very hard to exchange particles between the bulk and the interface. The interesting result here is that this process is inhomogeneous: it is faster at the topmost region of the bulk (just below the dense layer) and slower at the bottom of the system [57], as seen in Fig. 5. The oddity comes from the fact that the compaction is faster where the density is higher, the region where one would expect a slower evolution and a smaller number of holes. Instead, the evolution is faster and the number of holes is higher. Finally, it should be remarked that the small positive gradient seen in the density profile is consistent with experimental results $[1,13]$, although its sign is model dependent [58].

In Fig. 6 the parametric plot of $v$ versus $\rho$ in the out-ofequilibrium, aging regime is shown for several vibrations $x$ along with the equilibrium curve. Notice that despite the fact of not being stationary, the points, for several times (but the same $x$ ), roughly fall on a master curve, while different vibrations no longer collapse onto the same curve. However, these $x$-dependent universal curves no longer correspond to the equilibrium one, and as the vibration increases, there is a drift toward the equilibrium curve. The two regions seen 


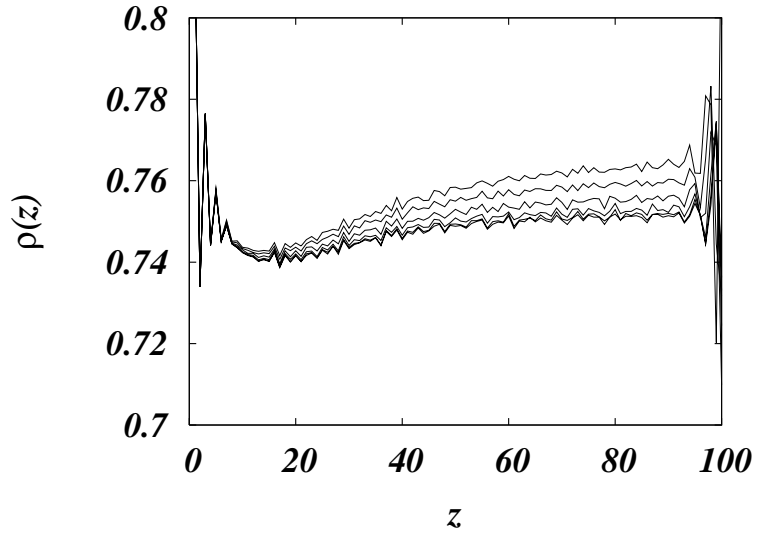

FIG. 5: Bulk density profile at different times (smaller time at the bottom). The region that compactifies the most is the denser one, close to the top, due to a larger number of holes.

in the $v$ profile, Fig. 4 , contribute differently for the curve in Fig. 6: the bulk forms the high $\rho$, small $v$ region near the horizontal axis (enlarged in the inset), while the interface generates the rest of the curve. In the inset of Fig. 6 we can see that the bulk behavior is the opposite of what would be expected at high densities: as the density increases the corresponding holes also increase! This explains why the density profile evolves faster near the dense layer where the density is higher: the greater the number of holes, the easier it is to compactify. Moreover, for different times, the curves no longer collapse (notice that the inset of Fig. 6 shows data for different times but same vibration $x$ ). Thus, differently from the holes from the interface, there is no universal curve for the holes in the bulk. Interestingly, besides having the opposite $\rho$ dependence, for larger times the curves move away from the equilibrium one (that is one order of magnitude above). Even the interface behavior is puzzling: layers whose density is small would be expected to be in local equilibrium. However, the hole distribution only coincides with the equilibrium one for $\rho \rightarrow 0$.

\section{CONCLUSIONS}

In summary, besides briefly reviewing the first application of the dynamical susceptibility $\chi_{4}$ in the context of granular compaction [37], we also extended the notion of dynamically available volumes for this externally driven, out of equilibrium situation. This is an additional similarity between structural glasses and granular systems, while shedding some light on the microscopic mechanisms responsible for the slow dynamics close to these transitions. Interestingly, the stationary behavior of the Kob-Andersen model with gravity is characterized by the local density, in spite of the macroscopically inhomogeneous density profile and the vibration imposed: layers with the same density presents the the same time dependence of $\chi_{4}(z, t)$ as well as the same density of holes. In the

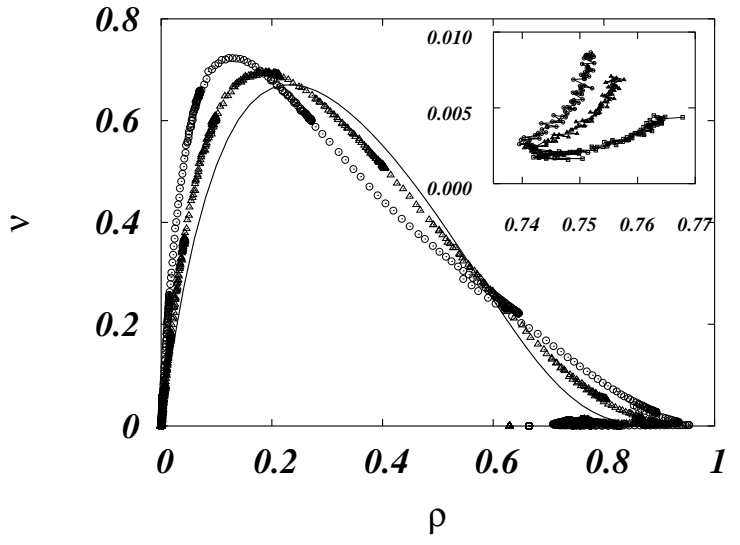

FIG. 6: Density of holes as a function of density for several values of $x$ in the aging regime (0.2, circle, and 0.4 , triangle), along with the equilibrium results for the KA model (line). Differently from the fluid phase, here there is no collapse of the curves onto the equilibrium curve. Notice that there are some points with $\rho>\rho_{c}$ that come from the oscillatory/dense layer regions. It is interesting to notice that in the case without gravity, the number of holes in the high- $\rho$ region is below the equilibrium one, while here it is above. Inset: Holes coming from the bulk for $x=0.2$ and three different times (from left to right: $t=1072,10974$ and 99999) as a function of density. Differently from what would be expected, this an increasing function of density. In these plots we do not take into account the oscillating layers near the bottom and near the dense layer, only the bulk layers $(6<z<95)$.

aging, compaction regime, this is no longer the case, although there is still a certain degree of universality in the behavior of the total number of holes from the interface, reflected on the collapse, at different times (but different densities), of all points onto a roughly universal curve that depends, in its turn, on the vibration (temperature). However, how connected and non-connected holes contribute to these profiles are yet to be investigated.

In addition, there are still many issues that deserve a closer inspection. Important information can also be obtained from the different sectors of the $\chi_{4}(t)$ function [36]. However, the range of time/density considered here is still small to resolve these different sectors. The distinction between connected and disconnected holes should also be made, as the former should be much more important in the compaction mechanism, along with the study of their spatial distribution [59]. Correlations may be also defined considering only the holes, with the associated dynamical susceptibility.

\section{Acknowledgments}

This work was partially supported by the Brazilian agencies CNPq, CAPES and FAPERGS. I acknowledge conversations with Mauro Sellitto, Yan Levin, Paolo di Gregorio and Aonghus Lawlor. Finally, I very much profited of the Abdus Salam ICTP (Italy) associationship support and kind environ- 
ment during the last years, where part of this research was carried on
[1] J. B. Knight, C. G. Fandrich, C. N. Lau, H. M. Jaeger, and S. R. Nagel, Phys. Rev. E 51, 3957 (1995).

[2] E. R. Nowak, J. B. Knight, M. L. Povinelli, H. M. Jaeger, and S. R. Nagel, Powder Technology 94, 79 (1997).

[3] A. Liu and S. Nagel, Nature 396, 21 (1998).

[4] E. R. Nowak, J. B. Knight, E. Ben-Naim, , H. M. Jaeger, and S. R. Nagel, Phys. Rev. E 57, 1971 (1998).

[5] J. Kurchan, in Jamming and Rheology: Constrained Dynamics on Microscopic and Macroscopic Scales, edited by S. F. Edwards, A. Liu, and S. R. Nagel (Taylor \& Francis, London, 1999), pp. 72-73, (cond-mat/9812347).

[6] C. Josserand, A. V. Tkachenko, D. M. Mueth, and H. M. Jaeger, Phys. Rev. Lett. 85, 3632 (2000).

[7] M. Nicolas, P. Duru, and O. Pouliquen, Eur. Phys. J. E 3, 309 (2000).

[8] A. Barrat, J. Kurchan, V. Loreto, and M. Sellitto, Phys. Rev. Lett. 85, 5034 (2000).

[9] C. S. O'Hern, S. A. Langer, A. J. Liu, and S. R. Nagel, Phys. Rev. Lett. 86, 111 (2001).

[10] G. D'Anna and G. Gremaud, Nature 413, 407 (2001).

[11] G. D'Anna and G. Gremaud, Phys. Rev. Lett. 87, 254302 (2001).

[12] L. Berthier, L. F. Cugliandolo, and J. L. Iguain, Phys. Rev. E 63, 051302 (2001)

[13] P. Philippe and D. Bideau, Europhys. Lett. 60, 677 (2002).

[14] L. E. Silbert, D. Ertas, G. S. Grest, T. C. Halsey, and D. Levine, Phys. Rev. E 65, 051307 (2002)

[15] H. A. Makse and J. Kurchan, Nature 415, 614 (2002).

[16] O. Pouliquen, M. Belzons, and M. Nicolas, Phys. Rev. Lett. 91, 014301 (2003).

[17] A. Kabla and G. Debrégeas, Phys. Rev. Lett. 92, 035501 (2004).

[18] G. Marty and O. Dauchot, Phys. Rev. Lett. 94, 015701 (2005).

[19] H. Makse, J. Brujić, and S. Edwards, cond-mat/0503081 (unpublished).

[20] O. Dauchot, G. Marty, and G. Biroli, Phys. Rev. Lett. 95, 265701 (2005)

[21] C. Song, P. Wang, and H. Makse, Proc. Nat. Acad. Sci. 102, 2299 (2005).

[22] M. Sellitto and J. J. Arenzon, Phys. Rev. E 62, 7793 (2000).

[23] A. Coniglio and H. Herrmann, Physica A 225, 1 (1995).

[24] J. J. Arenzon, J. Phys. A 32, L107 (1999).

[25] D. I. Goldman and H. L. Swinney, Phys. Rev. Lett. 96, 145702 (2006).

[26] H. Sillescu, J. of Non-Cryst. Sol. 243, 81 (1999).

[27] M. D. Ediger, Annu. Rev. Phys. Chem. 51, 99 (2000).

[28] R. Richert, J. Phys.: Condens. Matter 14, R703 (2002).

[29] H. Andersen, Proc. Nat. Acad. Sci. 102, 6686 (2005).

[30] R. Yamamoto and A. Onuki, Phys.Rev.Lett. 81, 4915 (1998).
[31] C. Bennemann, C. Donati, J. Baschnagel, and S. C. Glotzer, Nature 399, 246 (1999).

[32] J. Garrahan and D. Chandler, Phys. Rev. Lett. 89, 035704 (2002).

[33] L. Berthier and J. Garrahan, J. Chem. Phys. 119, 4367 (2003).

[34] L. Berthier, Phys. Rev. E 69, 020201(R) (2004).

[35] G. Biroli and J.-P. Bouchaud, Europhys. Lett. 67, 21 (2004).

[36] C. Toninelli, M. Wyart, L. Berthier, G. Biroli, and J.-P. Bouchaud, Phys. Rev. E 71, 041505 (2005).

[37] J. J. Arenzon, Y. Levin, and M. Sellitto, Physica A 325, 371 (2003).

[38] F. Ritort and P. Sollich, Adv. Phys. 52, 219 (2003).

[39] J. A. Drocco, M. B. Hastings, C. J. O. Reichhardt, and C. Reichhardt, Phys. Rev. Lett. 95, 088001 (2005).

[40] A. Lefèvre, L. Berthier, and R. Stinchcombe, Phys. Rev. E 72, 010301(R) (2005).

[41] L. E. Silbert, A. J. Liu, and S. R. Nagel, Phys. Rev. Lett. 95, 098301 (2005).

[42] A. Mehta and G. C. Barker, J. Phys. C 12, 6619 (2000).

[43] A. Lawlor, D. Reagan, G. D. McCullagh, P. D. Gregorio, P. Tartaglia, and K. Dawson, Phys. Rev. Lett. 89, 245503 (2002).

[44] T. Boutreux and P.-G. de Gennes, Physica A 244, 59 (1997).

[45] P. de Gregorio, A. Lawlor, P. Bradley, and K. Dawson, Phys. Rev. Lett. 93, 025501 (2004)

[46] P. de Gregorio, A. Lawlor, P. Bradley, and K. Dawson, Proc. Nat. Acad. Sci. 102, 5669 (2005).

[47] J. Jäckle and A. Krönig, J. Phys.: Condens. Matt. 6, 7633 (1994)

[48] C. Toninelli, G. Biroli, and D. Fischer, Phys. Rev. Lett. 92, 185504 (2004).

[49] C. Toninelli, G. Biroli, and D. S. Fisher, J. Stat. Phys. 120, 167 (2005).

[50] P. Richard, P. Philippe, F. Barbe, S. Bourlès, X. Thibault, and D. Bideau, Phys. Rev. E 68, 020301(R) (2003).

[51] K. Kob and H. Andersen, Phys. Rev. E 48, 4364 (1993).

[52] A. Imparato and L. Peliti, Phys. Lett. A 269, 154 (2000).

[53] L. Peliti and M. Sellitto, J. Phys. IV France 8, Pr6 (1998).

[54] Y. Levin, J. J. Arenzon, and M. Sellitto, Europhys. Lett. 55, 767 (2001).

[55] S. Franz, R. Mulet, and G. Parisi, Phys. Rev. E 65, 021506 (2002).

[56] J. J. Arenzon and M. Sellitto, Eur. Phys. J. B 42, 543 (2004).

[57] M. Sellitto, Phys. Rev. E 66, 042101 (2002).

[58] P. Philippe and D. Bideau, Phys. Rev. E 63, 051304 (2001).

[59] A. Lawlor, P. de Gregorio, P. Bradley, M. Sellitto, and K. Dawson, Phys. Rev. E 72, 021401 (2005). 